\title{
Late-diagnosed phenylketonuria in an eight-year-old boy with dyslexia and attention-deficit hyperactivity disorder
}

\author{
Yılmaz Yıldız', Ali Dursun ${ }^{1}$, Ayşegül Tokatlı1, Turgay Coşkun ${ }^{1}$, Hatice Serap Sivri ${ }^{1}$ \\ ${ }^{1}$ Pediatric Metabolic Diseases Unit, Department of Pediatrics, Hacettepe University Faculy of Medicine, Ankara, Turkey. \\ E-mail address: ssivri@hacettepe.edu.tr \\ Received: 22 March 2015, Revised: 10 April 2015, Accepted: 12 May 2015
}

\begin{abstract}
SUMMARY: Yıldız Y, Dursun A, Tokatlı A, Coşkun T, Sivri HS. Late-diagnosed phenylketonuria in an eight-year-old boy with dyslexia and attention-deficit hyperactivity disorder. Turk J Pediatr 2016; 58: 94-96.

Phenylketonuria, previously a common cause of severe intellectual disability, is a metabolic disorder now promptly diagnosed and effectively treated thanks to newborn screening programs. Here, we report a male patient presenting with dyslexia and attention-deficit hyperactivity disorder, who was diagnosed with mild phenylketonuria at eight years of age. Earlier recognition and treatment before the establishment of irreversible brain damage would have resulted in better neurobehavioural outcomes. Classical phenylketonuria and milder phenotypes of phenylalanine hydroxylase deficiency need to be considered in the differential diagnosis of all cognitive and behavioural problems of unknown cause.
\end{abstract}

Key words: phenylketonuria, phenylalanine hydroxylase deficiency, dyslexia, attentiondeficit hyperactivity disorder, intellectual disability.

Phenylalanine hydroxylase (PAH) deficiency, traditionally known as phenylketonuria (PKU), is one of the most common inherited disorders of amino acid metabolism and a leading cause of intellectual disability worldwide ${ }^{1}$. PAH deficiency encompasses a wide spectrum of phenotypes, ranging from mild hyperphenylalaninemia (HPA) not requring treatment to classical PKU, which may lead to severe developmental delay, intellectual disability, seizures and numerous neurobehavioural problems if treatment is not effectively initiated soon after birth ${ }^{2}$. Milder forms may present as more subtle neurocognitive deficits ${ }^{1}$, which may therefore be more difficult to diagnose. Newborn screening is crucial for prompt diagnosis and treatment ${ }^{3}$, but especially mild cases may be missed if the patient was not screened as a newborn. Here, we present a case of PAH deficiency with an atypical phenotype diagnosed at the age of eight.

\section{Case Report}

The patient came to attention when his newborn brother, a thirteen-day-old boy (A.H.) was referred to our clinic in Ankara, Turkey by his primary care provider upon positive newborn screening test for PAH deficiency (OMIM \#211600). His blood phenylalanine level was measured as $669 \mu \mathrm{mol} / \mathrm{L}(11.15 \mathrm{mg} /$ dl) and he was diagnosed with mild $\mathrm{PKU}^{2}$. His perinatal history and physical examination were unremarkable. Family history revealed non-consanguineous parents, a distant relative with classical PKU and an eight-year-old brother (M.H.) who had been diagnosed with dyslexia and attention-deficit hyperactivity disorder. A.H. was started on a phenylalanine-restricted diet and M.H. was summoned to our clinic for evaluation. M.H. also had a normal perinatal history and physical examination. He had no auditory or visual deficits. When he started elementary school, his teacher had noticed that his concentration in class was poor, he was exhibiting hyperactive behaviour, his reading ability was worse than his peers and he had difficulty in writing and reading certain letters. Wechsler Intelligence Scale for Children-IV (WISC-IV) was performed and he was found to have a normal intelligence quotient (IQ) of 97 . He was diagnosed with dyslexia and attention-deficit hyperactivity disorder based on the Diagnostic and Statistical Manual-IV 
(DSM-IV) criteria by the child and adolescent mental health specialist at his local hospital and was started on methylphenidate, but his response to medication was unsatisfactory. $\mathrm{He}$ had never been tested for PAH deficiency or received phenylalanine-restricted diet. His blood phenylalanine level was measured in our clinic and found to be $860 \mu \mathrm{mol} / \mathrm{L}(14.33 \mathrm{mg} / \mathrm{dl})$, also consistent with mild $\mathrm{PKU}^{2}$. Sequencing of PAH gene (OMIM *612349) in both brothers relevealed compound heterozygous mutations: c.1066-11G > A (IVS10-11G >A) and c.721C > T (p.Arg241Cys), the latter mutation predicting a tetrahydrobiopterin $\left(\mathrm{BH}_{4}\right)$-responsive phenotype of $\mathrm{PAH}$ deficiency ${ }^{4}$. $\mathrm{BH}_{4}$ loading test confirmed this prediction and sapropterin dihydrochloride treatment was initiated. Currently, both A.H. and M.H. have satisfactory metabolic control with pharmacotherapy alone for mild PKU.

\section{Discussion}

Newborn screening data show that the overall incidence of PKU is about 1 in 10000 live births in Europe, but varies from 1:30000 to $1: 3000^{3}$. Thanks to the advent of phenylalaninerestricted diet and implementation of newborn screening programs, severe developmental delay and intellectual disability secondary to PAH deficiency is now extremely rare in children of industrialized countries ${ }^{1}$. PAH deficiency is most commonly classified according to the severity of HPA before the initiation of treatment: Blood phenylalanine levels of 120-360 $\mu \mathrm{mol} / \mathrm{L}(2-6$ $\mathrm{mg} / \mathrm{dl}$ ) are defined as mild HPA not requring treatment, $360-600 \mu \mathrm{mol} / \mathrm{L}(6-10 \mathrm{mg} / \mathrm{dl})$ as mild HPA-gray zone (in reference to the disagreement about the need to treat individuals in this group), $600-900 \mu \mathrm{mol} / \mathrm{L}(10-15 \mathrm{mg} / \mathrm{dl})$ as mild PKU, $900-1200 \mu \mathrm{mol} / \mathrm{L}(15-20 \mathrm{mg} / \mathrm{dl})$ as moderate PKU and $>1200 \mu \mathrm{mol} / \mathrm{L}$ (>20 mg/ dl) as classical $\mathrm{PKU}^{2}$. Patients responding to pharmacological doses of $\mathrm{BH}_{4}$, the cofactor of the $\mathrm{PAH}$ enzyme, are defined as having $\mathrm{BH}_{4}{ }^{-}$ responsive $\mathrm{PAH}$ deficiency ${ }^{1}$. $\mathrm{PAH}$ deficiency differs from most other causes of intellectual disability by the availability of an effective treatment. Treatment is unequivocally indicated as soon as possible for blood phenylalanine levels above $600 \mu \mathrm{mol} / \mathrm{L}(10 \mathrm{mg} / \mathrm{dl})$ in order to prevent irreversible brain damage due to accumulation of phenylalanine ${ }^{5}$.

Clinical manifestations of untreated classical
PKU are now rarely encountered in children and adolescents, but include severe intellectual disability, microcephaly, convulsions, ataxia, autistic symptoms and various behavioural disturbances, such as self-mutilation, aggression, impulsivity and psychosis ${ }^{1}$. The severity of the clinical manifestations correlates with mean lifetime blood phenylalanine levels; with every $100 \mu \mathrm{mol} / \mathrm{L}(1.67 \mathrm{mg} / \mathrm{dl})$ increase above the normal range resulting in an estimated reduction of 1.9-4.1 IQ points ${ }^{6}$. Consequently, milder forms of PAH deficiency may have normal intelligence and development, but can present with more subtle symptoms like learning disabilities, mild behavioural disturbances and school problems in childhood or reduced emotional wellbeing, impaired ability to communicate and poor social functioning in adolescence and adulthood ${ }^{7}$. Children may be misdiagnosed as attention-deficit hyperactivity disorder or autism spectrum disorder and prescribed neurotropic drugs with no benefit; adolescents and adults may undergo extensive evaluations for leukodystrophy due to the typical brain magnetic resonance imaging findings of $\mathrm{PAH}$ deficiency ${ }^{8,9}$. Inability to diagnose mild forms of PAH deficiency poses additional harm in females, since high blood phenylalanine levels during pregnancy may result in developmental delay, intellectual disability, microcephaly, intrauterine growth retardation, congenital heart defects and other anomalies in the offspring, a teratogenic condition known as "maternal PKU syndrome"10. Also of note, the authors of this report have been unable to find previous reports of dyslexia in individuals with PAH deficiency.

PAH deficiency is an autosomal recessive trait. Its incidence in Turkey is approximately 1 in 4500 live births, with high rates of consanguinous marriages being a major contributory factor to this high incidence ${ }^{11}$. Newborn screening for PAH deficiency was initiated in Turkey in 1983 as a pilot project in a single center, and now has nationwide coverage. Our patient, M.H. was born in 2006, when national newborn screening coverage rate was $86.3 \%^{12}$. The chance of diagnosis was unfortunately missed until his brother was born eight years later.

It is important to consider PAH deficiency and to rule it out by performing basic plasma and 
urine amino acid analyses in individuals with autism spectrum disorders, attention-deficit hyperactivity disorders, learning difficulties and any cognitive or behavioural problem of unknown cause, especially if the individual was born before the initiation of newborn screening program or comes from a region where an efficient newborn screening program is unavailable ${ }^{1,13}$.

\section{REFERENCES}

1. Blau N, Burton BK, Thöny B, et al. Phenylketonuria and BH4 Deficiencies (1st ed). Bremen: UNI-MED Verlag AG; 2010.

2. Camp KM, Parisi MA, Acosta PB, et al. Phenylketonuria scientific review conference: state of the science and future research needs. Mol Genet Metab 2014; 112: 87-122.

3. Loeber JG. Neonatal screening in Europe; the situation in 2004. J Inherit Metab Dis 2007; 30: 430-438.

4. Anjema K, van Rijn M, Hofstede FC, et al. Tetrahydrobiopterin responsiveness in phenylketonuria: prediction with the 48-hour loading test and genotype. Orphanet J Rare Dis 2013; 8: 103-111.
5. Vockley J, Andersson HC, Antshel KM, et al. Phenylalanine hydroxylase deficiency: diagnosis and management guideline. Genet Med 2014; 16:188-200.

6. Waisbren SE, Noel K, Fahrbach K, et al. Phenylalanine blood levels and clinical outcomes in phenylketonuria: a systematic literature review and meta-analysis. Mol Genet Metab 2007; 92: 63-70.

7. Blau N, van Spronsen FJ, Levy HL. Phenylketonuria Lancet 2010; 376: 1417-1427.

8. Liu X, Guo H, Dahal M, Shi B. Unusual presentation of two Chinese phenylketonuria sisters who were misdiagnosed for years. BMJ Case Rep 2013.

9. Spilioti M, Evangeliou AE, Tramma D, et al. Evidence for treatable inborn errors of metabolism in a cohort of 187 Greek patients with autism spectrum disorder (ASD). Front Hum Neurosci 2013; 7: 858-684.

10. Saal HM, Braddock SR, Bull MJ, et al. Maternal phenylketonuria. Pediatrics 2008; 122: 445-449.

11. Ozalp I, Coskun T, Tokatli A, et al. Newborn PKU screening in Turkey: at present and organization for future. Turk J Pediatr 2001; 43: 97-101.

12. Tezel B, Dilli D, Bolat H, et al. The development and organization of newborn screening programs in Turkey. J Clin Lab Anal 2014; 28: 63-69.

13. Narayanan D, Barski R, Henderson MJ, et al. Delayed diagnosis of phenylketonuria - a case report of two siblings. Ann Clin Biochem 2014; 51: 406-408. 